\title{
A dynamical characterization of the small world phase
}

\author{
Tanya Araújo*, R. Vilela Mendes ${ }^{\dagger \dagger}$, João Seixas ${ }^{\S}$
}

\begin{abstract}
Small-world (SW) networks have been identified in many different fields. Topological coefficients like the clustering coefficient and the characteristic path length have been used in the past for a qualitative characterization of these networks. Here a dynamical approach is used to characterize the small-world phenomenon. Using the $\beta$-model, a coupled map dynamical system is defined on the network. Entrance to and exit from the SW phase are related to the behavior of the ergodic invariants of the dynamics.
\end{abstract}

Keywords: Small World, Ergodic invariants, Coupled maps, Phase transitions

\section{Introduction}

Networks are prevalent in all domains of life and science. Social, economic and political networks are the backbone of human society. The internet is a network. The metabolic processes of living beings form a network with the

\footnotetext{
${ }^{*}$ Dept. Economia, UECE, ISEG, R. Miguel Lupi 20, 1200 Lisboa, Portugal (tanya@iseg.utl.pt)

${ }^{\dagger}$ corresponding author

${ }^{\ddagger}$ Grupo de Física-Matemática, Complexo Interdisciplinar, Universidade de Lisboa, Av. Gama Pinto 2, 1699 Lisboa Codex, Portugal (vilela@cii.fc.ul.pt)

${ }^{\S}$ Departamento de Física, Instituto Superior Técnico, Av. Rovisco Pais, 1096 Lisboa Codex, Portugal (seixas@fisica.ist.utl.pt)
} 
substrates as nodes, which are linked whenever they participate in the same biochemical reaction. Protein-protein and gene expression and regulation are biological networks, etc.

Regular lattices and random graphs [1] have for a long time been studied. More recently [2]-[3] small-world networks became the object of growing attention and were identified in many different fields. They seem to be the underling structure for some important phenomena like the rapid spread of diseases, social networks, cooperative behavior between competing agents, problem solving organization and communication networks.

Topologically, small-world (SW) networks are identified by the values of two statistical properties:

- the clustering coefficient $(C C)$ that measures the average probability for two agents, having a common neighbor, to be themselves connected and

- the characteristic path length $(P L)$, this being the average length of the shortest path connecting each pair of agents.

Regular lattices have long path lengths and high clustering, whereas random graphs have short path lengths but low clustering. SW networks exhibit short $P L$ 's and, at the same time, high $C C^{\prime}$ s.

In many model networks, the simultaneous occurrence of high $C C$ and low $P L$ is observed over an interval between order and randomness, which is called the SW phase. However, this phenomenon can only be defined as a phase, in the statistical mechanics sense, if order parameters are found to characterize the regular-to-SW and the SW-to-random phase transitions.

Further information on the SW phenomenon has been obtained in the past from the study of several quantities. Farkas et al. [4] studied the spectral density of the adjacency matrix, with increasing randomness, concluding that, in spite of the blurring of singularities, a consistently high value of the third moment implies the existence of a large number of triangles in the SW network. Monasson [5] on the other hand studied the spectral properties of the Laplacian operator, that characterizes the time evolution of a diffusive field, and localization properties on the graph.

In this paper a dynamical systems approach is used to characterize the small-world phenomenon. Using the $\beta$-model[3], we study a coupled map 
system on the network, with interactions defined by the network connections. The SW phase is related to the behavior of the ergodic invariants of the dynamics. Entrance to the SW phase is related to the Lyapunov spectrum and exit from the SW phase corresponds to the region where "entropy" and "conditional exponents entropy" [6] [7] split apart.

\section{The dynamical model}

Consider a $\beta$-family of models, each one with $N$ agents on a circle and periodic boundary conditions. For $\beta=0$, each agent in the model is connected to its $2 v$ nearest neighbors. For $\beta \neq 0$, the network structure is obtained by looking at each one of the connections of the $\beta=0$ structure and, with probability $\beta$, replacing this connection by a new random one.

On each one of the $\beta$-networks, a dynamical system is defined, with a map at each node and convex-coupling interactions defined by the network connections

$$
x_{i}(t+1)=\sum_{j=1}^{N} W_{i j} f\left(x_{j}(t)\right)
$$

where

$$
W_{i j}= \begin{cases}1-\frac{n_{v}(i)}{2 v} c & \text { if } i=j \\ \frac{c}{2 v} & \text { if } i \neq j \text { and } i \text { is connected to } j \\ 0 & \text { otherwise }\end{cases}
$$

$n_{v}(i)$ is the number of agents connected to $i$ and $\mathrm{c}$ is a control parameter.

For the agents dynamics we choose

$$
f(x)=\alpha x \quad \text { mod. } 1
$$

Typically $\alpha=2$.

For the $\beta=0$ network, each agent has exactly $2 v$ neighbors and the Lyapunov exponents are

$$
\lambda_{0}(k)=\log \left\{\alpha\left(1-c+\frac{c}{v} \sum_{j=1}^{v} \cos \left(j \theta_{k}\right)\right)\right\}
$$

with $\theta_{k}=\frac{2 \pi k}{N}, k=0, \cdots, N-1$. In the $N \rightarrow \infty$ limit, the Lyapunov spectrum is a continuous smooth function, as illustrated in the upper plot of 

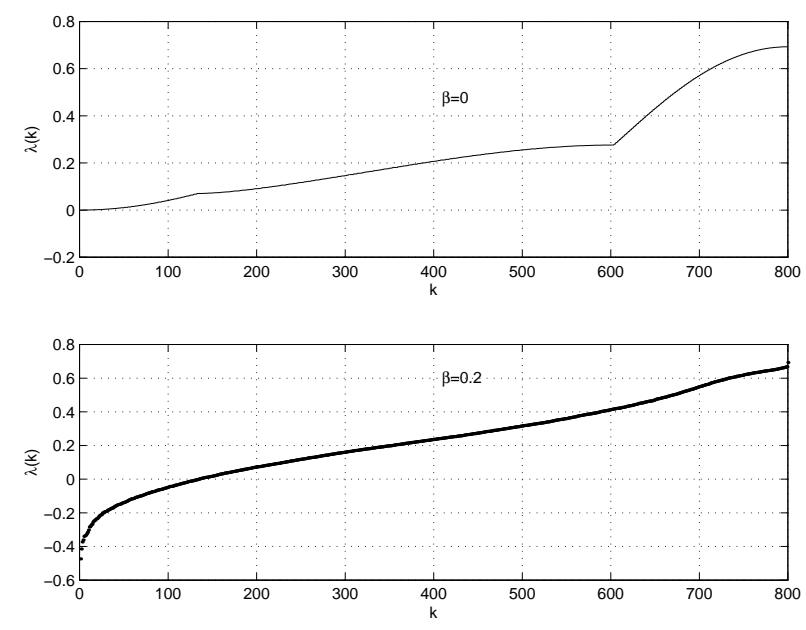

Figure 1: Lyapunov spectrum for $\beta=0$ and for a typical network at $\beta=0.2$ $(N=800,2 v=6)$

Fig. 1. For reasons to be discussed below we always choose $c$ in such a way that, for $\beta=0$, the lowest Lyapunov exponent is zero.

As $\beta$ increases, the matrices of the tangent map cease to be regularly organized, the Lyapunov spectrum develops gaps and some of the exponents become negative. This is illustrated in the lower plot of Fig. 1 for $N=800$ and $2 v=6$.

It is also the appearance of random long range connections that is responsible for the reduction of the path length in SW networks. Therefore it is natural to consider the modifications in the Lyapunov spectrum as the dynamical signature of the onset of the SW phase. Of particular dynamical significance is the shift of part of the spectrum towards negative values. That is, the randomness arising from the rewiring leads to an effective reduction of the dynamical degrees of freedom. We define $D_{\beta}$

$$
D_{\beta}=-\sum_{\lambda_{i}<0} \lambda_{i}
$$

to quantify this effect. To characterize the modifications of the Lyapunov spectrum, another possibility would be to measure the singular part of the 
spectrum associated to the gaps. However the natural intervals in the spectrum associated to finite $N$ make this measurement less reliable.

In the upper plot of Fig. 2 we show the average values of $D_{\beta}$ taken over 100 different samples for each $\beta$ (with $N=800$ and 6 as the average degree of the network). A good fit to all the data shown in the lower plot of Fig.2 is

$$
D_{\beta}=c N\left(\beta-\beta_{c_{1}}\right)^{\eta_{1}}
$$

with $\beta_{c_{1}} \leq 10^{-5}$ and $\eta_{1}=1.01 \pm 0.06$.
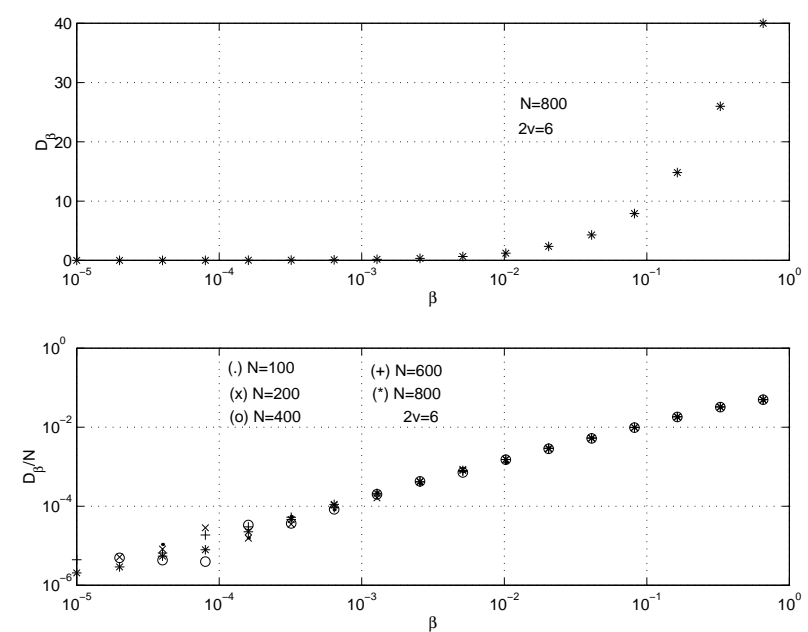

Figure 2: The $D_{\beta}$ parameter ("SW order parameter") averaged over 100 sample networks $(N=100, \cdots, 800,2 v=6)$

In practice it is only after $\beta \simeq 10^{-3}$ that small-world effects (and $D_{\beta}$ values) become appreciable. Nevertheless, the fact that the data is consistent with $\beta_{c_{1}}=0$ implies that, using $\frac{1}{N} D_{\beta}$ as an order parameter for the smallworld phase, this phase starts at $\beta=0^{+}$, the regular phase being only the isolated point $\beta=0$.

To characterize the exit from the SW phase, we use the notion of conditional Lyapunov exponents. They were introduced by Pecora and Carroll in their study of synchronization of chaotic systems [8]. Like the Lyapunov exponents, the conditional exponents are well defined ergodic invariants [6]. 
The idea is that the conditions that in Oseledec's theorem insure the existence of the Lyapunov exponents also establish the existence of characteristic exponents formed by subblocks of the tangent map matrix. Here, for each agent $i$, we consider a subblock of dimension $d_{i} \times d_{i}$ formed by himself and those that are connected to it. The positive conditional exponents $\lambda_{\beta}^{*}(j)$ associated to each subblock are computed and a dimension-weighed sum is performed over all subblocks. This gives a version of what elsewhere[6] [7] has been called a conditional exponents entropy.

$$
h_{\beta}^{*}=\sum_{i=1}^{N}\left(\frac{1}{d_{i}} \sum_{\lambda_{\beta}^{*}>0} \lambda_{\beta}^{*}(j)\right)
$$

Subtracting $h_{\beta}^{*}$ from the sum of the positive Lyapunov exponents, $h_{\beta}=$ $\sum_{\lambda_{\beta}>0} \lambda_{\beta}(j)$, we define the coefficient

$$
C_{\beta}=\left|\frac{h_{0}^{*}-h_{0}}{h_{\beta}^{*}-h_{\beta}}\right|
$$

which is also an ergodic invariant.

This coefficient has the following dynamical interpretation: The Lyapunov exponents measure the rate of information production or, from an alternative point of view, they define the dynamical freedom of the system, in the sense that they control the amount of change that is needed today to have an effect on the future. In this sense the larger a Lyapunov exponent is, the freer the system is in that particular direction, because a very small change in the present state will induce a large change in the future. The conditional exponents have a similar interpretation concerning the dynamics as seen from the point of view of each agent and his neighborhood [7]. However the actual information production rate is given by the sum of the positive Lyapunov exponents, not by the sum of the conditional exponents. Therefore, the quantity $h_{\beta}^{*}-h_{\beta}$ is a measure of apparent dynamical freedom (or apparent rate of information production). As self-organization in a system concerns the dynamical relation of the whole to its parts, this quantity may also be looked at as a measure of dynamical selforganization.

In Fig. 3 we show the average values of $C_{\beta}$ taken over 100 different samples for each $\beta$ (with 6 as the average degree of the network and $N=$ $100,200,400,600,800)$. Notice the $N$-independence of $C_{\beta}$ which follows from 


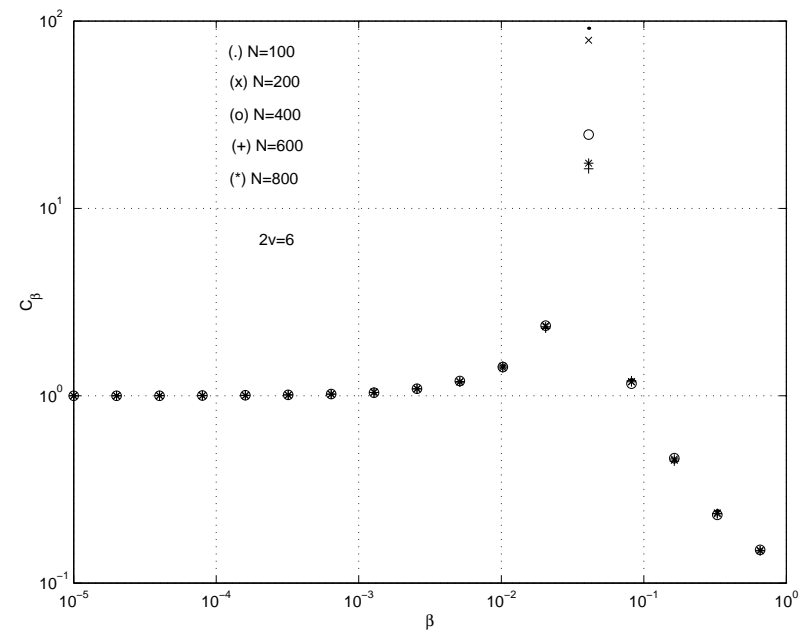

Figure 3: The $C_{\beta}$ parameter ("SW exit") averaged over 100 sample networks $(N=100, \cdots, 800,2 v=6)$

the fact that, in Eq.(8) it is defined as a ratio of two quantities with the same $N$-dependence. For small $\beta$ values the difference between the entropy and the conditional exponents entropy is a small quantity, that may be easily computed from the network parameters. It means that each agent may have exact information on the global behavior from observation of his own neighborhood. When $\beta$ increases the difference changes sign and becomes very large meaning that the neighborhood information has ceased to provide reliable information on the actual dynamics. This is the dynamical correlate of the decreasing cluster properties and allows us to define the transition at the divergence point $\beta_{c_{2}}$ of $C_{\beta}$. We find

$$
\beta_{c_{2}} \simeq 0.04
$$

Near the transition region

$$
C_{\beta} \sim\left|\beta-\beta_{c_{2}}\right|^{-\eta_{2}}
$$

with $\eta_{2} \simeq 1.14$ below the transition and $\eta_{2} \simeq 0.93$ above it. 


\section{Conclusions}

1 - The ergodic invariants (Lyapunov spectrum and conditional exponents) provide a link between the topological properties of SW networks and the dynamical behavior of a coupled map system modelled on the network. In addition, the power laws obeyed by these invariants provide a framework to identify the SW phenomenon as a phase in the statistical mechanics sense.

2 - Coupled map behavior, evolution of a diffusive field [5] and spectrum of the adjacency matrix 团 supply complementary information on the SW phenomenon. It is therefore conceivable that quantities obtained from these other approaches might also be used to construct order parameters characterizing the SW phase.

\section{References}

[1] P. Erdös and A. Rényi; Publ. Math. Inst. Hung. Acad. Sci. 5 (1960) 17.

[2] D. J. Watts and S. H. Strogatz; Nature 393 (1998) 440.

[3] D. J. Watts; Small Worlds, Princeton Univ. Press, Princeton 1999.

[4] I. J. Farkas, I. Derényi, A.-L. Barabási and T. Viczek; Phys. Rev. E64 (2001) 026704.

[5] R. Monasson; Europhys. J. B12 (2000) 555.

[6] R. Vilela Mendes; Phys. Lett. A248 (1998) 167.

[7] R. Vilela Mendes; Physica A 276 (2000) 550, A 295 (2001) 537.

[8] L. M. Pecora and T. L. Carroll; Phys. Rev. Lett. 64 (1990) 821; Phys. Rev. A44 (1991) 2374. 